\title{
Expanding Hermitian operators in a basis of projectors on coherent spin states
}

\author{
Stefan Weigert \\ $\langle\mathrm{Hu} \mid \mathrm{MP}\rangle$-Hull Mathematical Physics, Department of Mathematics, University of Hull, \\ Hull HU6 7RX, UK \\ E-mail: S.Weigert@hull.ac.uk
}

Received 1 September 2004, accepted for publication 7 October 2004

Published 15 October 2004

Online at stacks.iop.org/JOptB/6/489

doi:10.1088/1464-4266/6/12/001

\begin{abstract}
The expectation values of a Hermitian operator $\hat{A}$ in $(2 s+1)^{2}$ specific coherent states of a spin are known to determine the operator unambiguously. As shown here, (almost) any other set of $(2 s+1)^{2}$ coherent state projectors also provide a basis for self-adjoint operators. This is proved by considering the determinant of the Gram matrix associated with the coherent state projectors as a Hamiltonian of a fictitious classical spin system. The result guarantees that (almost) any experimentally desirable choice of directions is appropriate for reconstructing the state of a quantum spin by means of a Stern-Gerlach apparatus.
\end{abstract}

Keywords: state reconstruction, quorum, coherent states, density matrix

State reconstruction [1] aims at parametrizing the density matrix $\hat{\rho}$ of a quantum system by the expectations of appropriately chosen observables, the quorum. For a spin $s$, the (unnormalized) density matrix has $N_{s}=(2 s+$ $1)^{2}$ independent real parameters, and various reconstruction schemes exist $[2,3]$.

In [4], a particularly simple and non-redundant quorum has been identified. It consists of precisely $N_{s}$ projectors on coherent spin states $|\mathbf{n}\rangle$, with $\mathbf{n} \cdot \hat{\mathbf{S}}|\mathbf{n}\rangle=\hbar s|\mathbf{n}\rangle$. The quorum is non-redundant in the sense that one cannot do with a smaller number of projectors. Indeed, the density matrix $\hat{\rho}$ of a spin $s$ is determined unambiguously if one performs the following measurements with a traditional SternGerlach apparatus. Consider $(2 s+1)$ cones about the $z$ axis with different opening angles. Then, pick $(2 s+1)$ directions on each cone such that the set transforms into itself under a rotation about $z$ by an angle $2 \pi /(2 s+1)$. This gives a total of $N_{s}$ directions $\mathbf{n}_{n}, n=1,2, \ldots, N_{s}$ evenly spaced on $(2 s+1)$ cones. Measuring the $(2 s+1)^{2}$ relative frequencies $p_{s}\left(\mathbf{n}_{n}\right)=\left\langle\mathbf{n}_{n}|\hat{\rho}| \mathbf{n}_{n}\right\rangle$ defines a unique (unnormalized) density matrix $\hat{\rho}$. The operator $\hat{\rho}$ is thus fixed by its expectation values in the coherent states $\left|\mathbf{n}_{n}\right\rangle$.

In fact, the result applies to any Hermitian operator $\hat{A} \in \mathcal{A}_{s}$, the space of linear operators acting in the Hilbert space $\mathcal{H}_{s}$ of the spin. The values of its $Q$-symbol, $Q_{A}(\mathbf{n})=$ $\operatorname{Tr}[\hat{A}|\mathbf{n}\rangle\langle\mathbf{n}|]=\langle\mathbf{n}|\hat{A}| \mathbf{n}\rangle$, at $N_{s}$ points corresponding to the set of directions described above determine the operator $\hat{A}$ unambiguously. In the following, let us denote a set of $N_{s}$ points (as well as the associated $N_{s}$ unit vectors $\mathbf{n}_{n}$ ) as a 'constellation' $\mathcal{N}$ or a 'hedgehog' $\mathcal{N}$ with unit spikes $\mathbf{n}_{n}$.

The spatial directions $\mathbf{n}_{n}$ dealt with in [4] were restricted to a certain class of regular hedgehogs, $\mathcal{N}_{0}$. The purpose of the present contribution is to remove this restriction. Given a generic constellation $\mathcal{M}$, the $N_{s}$ values of the $Q$-symbol $Q_{A}\left(\mathbf{n}_{n}\right)$ will (almost always) contain all the information about the operator $\hat{A}$. More precisely: given any constellation $\mathcal{M}$ of vectors $\mathbf{m}_{n}$, then either the numbers $Q_{A}\left(\mathbf{m}_{n}\right)$ determine $\hat{A}$, or there is an infinitesimally close constellation $\mathcal{M}^{\prime}$ such that the numbers $Q_{A}\left(\mathbf{m}_{n}^{\prime}\right)$ can be used instead. Two constellations $\mathcal{M}$ and $\mathcal{M}^{\prime}$ are close if, for example, the number

$$
d\left(\mathcal{M}, \mathcal{M}^{\prime}\right)=\sum_{n=1}^{N_{s}}\left|\mathbf{m}_{n}-\mathbf{m}_{n}^{\prime}\right|
$$

is small. Here is a low-dimensional example which illustrates the 'almost always' aspect of the statement made above. Consider the real vector space $\mathbb{R}^{3}$ : any three unit vectors attached to the origin are a basis as long they are neither in a plane nor parallel to each other. Clearly, the exceptional cases have measure zero among all possibilities: picking three directions at random, the corresponding vectors will span $\mathbb{R}^{3}$ with probability one. Furthermore, there are always three linearly independent vectors arbitrarily close to each exceptional situation. 
Let us now turn to the proof. Consider the $N_{s}$ projection operators on coherent states,

$$
\hat{Q}_{n}=\left|\mathbf{n}_{n}\right\rangle\left\langle\mathbf{n}_{n}\right|, \quad \mathbf{n}_{n} \in \mathcal{N}_{0}, \quad 1 \leqslant n \leqslant N_{s},
$$

associated with a constellation $\mathcal{N}_{0}$ of the type given in [4]. The following argument shows that the projectors $\hat{Q}_{m}, m=$ $1,2, \ldots, N_{s}$, associated with any other hedgehog $\mathcal{M}$ (or $\mathcal{M}^{\prime}$, infinitesimally close to $\mathcal{M}$ ) span the space $\mathcal{A}_{s}$. The $N_{s}^{2}$ elements of the Gram matrix $\mathrm{G}_{n n^{\prime}}$ [5] determined by a constellation $\mathcal{M}$ are given by the scalar products of the projection operators:

$$
\begin{aligned}
\mathrm{G}_{n n^{\prime}} & =\operatorname{Tr}\left[\hat{Q}_{n} \hat{Q}_{n^{\prime}}\right]=\left|\left\langle\mathbf{m}_{n} \mid \mathbf{m}_{n^{\prime}}\right\rangle\right|^{2}=\left(\frac{1+\mathbf{m}_{n} \cdot \mathbf{m}_{n^{\prime}}}{2}\right)^{2 s}, \\
1 & \leqslant n, n^{\prime} \leqslant N_{s} .
\end{aligned}
$$

The result in [4] essentially says that the Gram matrix stemming from the constellation $\mathcal{N}_{0}$ has a non-zero determinant. According to (3), the matrix elements of $G$ are polynomial expressions in the components of the unit vectors $\mathbf{m}_{n}$ and $\mathbf{m}_{n^{\prime}}$. Thus, the determinant of the matrix $\mathbf{G}$ is also a polynomial in these components, and the derivatives of $\operatorname{det} \mathrm{G}$ exist to arbitrary order. The determinant of $\mathrm{G}$ is a (real) analytic function of each spin component.

Consider now a constellation $\mathcal{N}_{0}$. When keeping the vectors $\mathbf{n}_{1}, \ldots, \mathbf{n}_{n-1}$ and $\mathbf{n}_{n+1}, \ldots, \mathbf{n}_{N_{s}}$ fixed, the function $\operatorname{det} \mathrm{G}$ can be regarded as a fictitious time-independent Hamiltonian $H_{n}$ of a single classical spin, $\mathbf{n}_{n}$ :

$$
H_{n}\left(\mathbf{n}_{n}\right)=\operatorname{det} \mathrm{G}\left(\mathbf{n}_{n}\right) .
$$

The Hamiltonian $H_{n}$ is different from zero if $\mathbf{n}_{n}$ coincides with the $n$th vector of the constellation $\mathcal{N}_{0}$. It describes an integrable system since there is just one degree of freedom accompanied by one constant of motion, the Hamiltonian itself [6]. Consequently, the two-dimensional phase space $\mathcal{S}^{2}$ is foliated by one-dimensional tori of constant energy. In addition, a finite number of (elliptic or hyperbolic) fixed points and one-dimensional separatrices will occur. These are the generic features of the flow generated by the Hamiltonian $H_{n}\left(\mathbf{n}_{n}\right)$ on the unit sphere $\mathcal{S}^{2}$ :

$$
\frac{\mathrm{d} \mathbf{n}_{n}}{\mathrm{~d} t}=\mathbf{n}_{n} \times \frac{\partial H}{\partial \mathbf{n}_{n}},
$$

where $\partial / \partial \mathbf{n}_{n}$ is the gradient with respect to $\mathbf{n}_{n}$ [7]. The right-hand-side being a (non-constant) polynomial in the components of $\mathbf{n}_{n}$ implies that the integral curves of the Hamiltonian are fixed points, separatrices, and closed orbits. Due to its analyticity properties, the function $H_{n}\left(\mathbf{n}_{n}\right)$ cannot take the value zero in a finite two-dimensional region-it would need to be identical to zero then. It will be zero at most for a finite number of one-dimensional curves or points. Consequently, the function $\operatorname{det} \mathrm{G}\left(\mathbf{n}_{n}\right)$ is different from zero for almost all choices of $\mathbf{n}_{n}$. It is thus possible to replace the vector $\mathbf{n}_{n}$ by any other vector, including $\mathbf{m}_{n}$, the $n$th vector of the desired constellation $\mathcal{M}$. If one follows a continuous path on $\mathcal{S}^{2}$ connecting $\mathbf{n}_{n}$ to $\mathbf{m}_{n}$, one will possibly pass through points where the determinant of $\mathrm{G}$ vanishes. If, accidentally, $\mathbf{m}_{n}$ were to correspond to a point with vanishing energy, there would always be a nearby vector $\mathbf{m}_{n}^{\prime}$ with $\left|\mathbf{m}_{n}^{\prime}-\mathbf{m}_{n}\right|<\varepsilon / N_{s}$ since levels of constant energy have co-dimension one.
If one repeats this procedure $N_{s}$ times, one ends up with a constellation $\mathcal{M}^{\prime}$ infinitesimally close to $\mathcal{M}$ since $\sum_{n} \mid \mathbf{m}_{n}-$ $\mathbf{m}_{n}^{\prime} \mid<\varepsilon$ can be made arbitrarily small. With probability one, the exact constellation $\mathcal{M}$ is obtained. Consequently, almost all hedgehogs $\mathcal{M}$ of $N_{s}$ projection operators $\hat{Q}_{n}$ give rise to a basis in the space of linear operators on $\mathcal{H}_{s}$, the Hilbert space of a spin $s$. In turn, the values of the discrete $Q$-symbol related to a constellation $\mathcal{M}$ are indeed sufficient to determine the operator $\hat{A}$.

The result obtained in this note has interesting implications from both a theoretical and an experimental point of view. On the one hand, it satisfactory to know that, in principle, (almost) any constellation $\mathcal{M}$ of $N_{s}$ directions can be used to reconstruct a quantum spin state, using an ordinary SternGerlach apparatus. On the other hand, imagine actually performing the reconstruction in the laboratory. Clearly, an experimenter would look for a constellation which is experimentally feasible (not all directions might be accessible), which provides the best signal-to-noise ratio (close directions should be avoided), and which uses the most efficient matrix inversion (the determinant of the Gram matrix should not be small). The present result, firstly, tells the experimenter that (almost) any feasible constellation can be used; secondly, the result allows the experimenter to opt for constellations in which the directions are as 'different' as possible from each otherideally, there will be only one direction inside any cone with opening angle $4 \pi / N_{s}$; thirdly, the experimenter should select those of the remaining desirable constellations which do not lead to small values of the determinant of $\mathrm{G}$ - the numerical implementation of the reconstruction will be most efficient then.

In summary, it has been shown that (almost) any distribution of $N_{s}$ points on a sphere $\mathcal{S}^{2}$ gives rise to a basis of (non-orthogonal) coherent state projectors $\hat{Q}_{n}$ in the linear space $\mathcal{A}_{s}$. As shown in [8], one can develop a fully fledged discrete equivalent of $Q$ - and $P$-symbols each consisting of $N_{s}$ operators. An interesting expectation-value representation of the dynamics of a quantum spin $s$ emerges from this approach [9], while the relation of the basis of projectors $\hat{Q}_{n}$ to a symbolic calculus à la Stratonovich and Weyl has been elaborated on in [10].

\section{Acknowledgment}

This work was supported partially by the Schweizerische Nationalfonds.

\section{References}

[1] Leonhardt U 1997 Measuring the Quantum State of Light (Cambridge: Cambridge University Press)

[2] Man'ko V I and Man'ko O V 1997 J. Exp. Theor. Phys. 85430

[3] Agarwal G S 1998 Phys. Rev. A 57671

[4] Amiet J-P and Weigert S 1999 J. Phys. A: Math. Gen. A 32 L 269

[5] Greub W H 1963 Linear Algebra (Berlin: Springer)

[6] Arnold V I 1984 Mathematical Methods of Classical Mechanics (Berlin: Springer)

[7] Srivastava N, Kaufman C, Müller G, Magyari E, Weber R and Thomas H 1987 J. Appl. Phys. 614438

[8] Amiet J P and Weigert S 2000 J. Opt. B: Quantum Semiclass. Opt. 2118

[9] Weigert S 2000 Phys. Rev. Lett. 84802

[10] Weigert S 1999 Acta Phys. Slovaca 49613 